\title{
Compiling method of automobile fault diagnosis information
}

\author{
Guofang $\mathrm{Wu}^{*}$, Fujia Liu, and Shuquan $\mathrm{Xu}$ \\ Research Institute of Highway Ministry of Transport, Beijing, China
}

\begin{abstract}
Automobile fault diagnosis is the most technical content in automobile maintenance. The speed, accuracy and convenience of diagnosis directly affect the maintenance efficiency. Automobile fault diagnosis information is an important reference for finding and solving automobile faults. High quality information is the key to improve technical personnel's judgment and solving faults. In this paper, the methods and examples of fault diagnosis information compilation are given to guide the technical data writers to compile effective fault diagnosis information for automobiles.
\end{abstract}

Keywords: Automobile, Fault, Diagnosis, Information.

\section{Introduction}

Automobile fault diagnosis refers to the detection, analysis and judgment to determine the technical condition of the automobile or find out the fault position and reason under the condition of not disassembling (or only disassembling individual parts) when the automobile has hidden trouble, the technical condition has become worse, or has partially or even completely lost the working ability.

Automobile fault diagnosis information is to collect fault phenomena, fault causes, fault diagnosis and detection methods, and to provide information on the analysis, judgment and inspection process of finding out the cause and position of fault. It is an important guidance information for finding and troubleshooting faults and restoring the technical condition and working ability of the car when it leaves the factory.

\section{Automobile fault classification}

There are two types of car failures. One is mechanical failure, which is caused by the mechanical parts themselves or the interaction and cooperation between several mechanical parts. Due to the natural wear, deformation, aging, damage, fatigue, corrosion and other causes of the automobile assembly or parts in the mechanism, the main performance is the sudden change of working conditions, abnormal sound, abnormal smell, abnormal smoke

*Corresponding author: gf.wu@rioh.cn 
exhaust, abnormal temperature, abnormal appearance, abnormal consumption of fuel and lubricating oil, leakage and so on[1]. The other is an electronic failure, which is caused by a problem with electronic equipment or parts. Generally due to damage of sensors, actuators, control units, plugins, wiring harness, etc. Through ECU analysis, OBD record information, diagnostic instrument read fault code to obtain vehicle performance and fault information parameters, compared with the normal vehicle technical conditions, analysis and judgment of technical performance and fault diagnosis conclusions[2].

\section{Current situation of fault diagnosis information compilation}

This paper selects and analyzes the fault diagnosis information of 108 models (including passenger cars, buses and trucks from Europe, America, Japan, South Korea and domestic independent brands in accordance with the principle that the sales volume occupies a large market share), and finds that the problems existing in the fault diagnosis data are mainly manifested in the following aspects:

1) Lack of partial assembly, system, parts of the fault phenomenon;

2) Lack of fault codes and definitions for all or part of the electronic control system;

3) Lack of fault diagnosis and troubleshooting methods and steps;

4) Missing parameter information for detection and troubleshooting.

The problem distribution is shown in Figure 1.

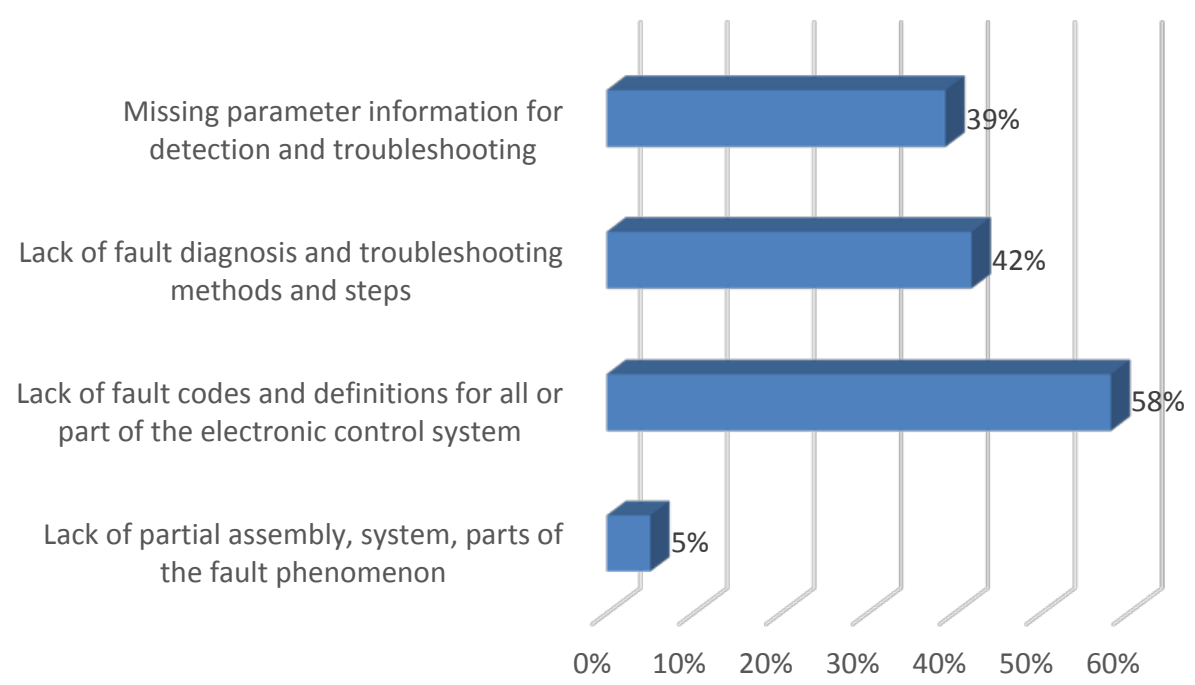

Fig. 1. Problems with the fault diagnosis information.

The comprehensive analysis may have the following reasons:

1) The vehicle structure was not clearly and systematically divided, and the necessary parts were selected according to the experience when constructing the data of different models;

2) The electronic control system components with fault codes are not fully grasped, and the fault codes and definition information of some electronic control systems are missing;

3) The importance of data compilation is not well understood, the system maintenance data provided by external suppliers is ignored, and the data of complete models is not combined; 
4) The technical documentation clerk did not simulate the fault and lacked practical experience in fault diagnosis and troubleshooting. The description of fault diagnosis and troubleshooting methods and steps was too concise or the relevant parameter information for detection and fault diagnosis was missing in the description process, which could not reach the level of guiding operation.

\section{Content of fault diagnosis information}

Fault diagnosis information ${ }^{[3]}$ mainly includes the following aspects:

1) Description of fault phenomena;

2) Fault diagnosis process;

3) Fault diagnosis and elimination methods;

4) Technical parameters, including data parameters and their meanings, and reasonable range of data parameter values;

5) Fault code and definition of electronic control system ${ }^{[4]}$.

\section{Requirements for compilation of fault diagnosis information}

\subsection{Language Expression}

Language expression should be concise, clear, accurate, without ambiguity.

Standardize the use of written technical terms.

The international system of units should be used in common use.

\subsection{Formal Expression}

Fault tree ${ }^{[5]}$ is the most common expression method to reflect the logical relationship between fault characteristics and fault causes. It is a qualitative causal model based on the structure and functional characteristics of the diagnosed object, and a kind of inverted tree structure with logic gate to represent the relationship between events. The phenomenon is complicated by the car breakdown. A failure phenomenon, there may be a variety of reasons; A single cause may lead to a variety of faults. Therefore, the establishment of a correct and reasonable fault tree is the core of successful fault diagnosis.

\subsection{Preparation Steps}

To write the fault tree, the first step is to determine the top event, which is the symptom description of the fault. The phenomenon can be described from the aspects of sound, smell, smoke exhaust, temperature, appearance and so on. Then, according to experience, according to the possibility of causing failure, a recommended elimination sequence is given, and the description of fault diagnosis and elimination method is given. It is necessary to explain the detection method, detection tool, detection preparation work, matters needing attention and other information, so as to clarify what maintenance personnel should do. Summarize complete and correct data according to vehicle simulation fault. After replacement of parts, confirm that the fault has been repaired. Detailed preparation can be done by referring to the following steps:

Step 1:Fault symptom description or fault code definition

Step 2:Possible causes of this failure 1

Step 3:Method and process for troubleshooting fault 1

Step 4 :Possible causes of this failure 2 
Step 5 :Method and process for troubleshooting fault 2

Step $6: \ldots . .$.

Step 7:Inspection of maintenance effect

\subsection{Information Verification}

In order to ensure the operability, accuracy, completeness and security of the fault diagnosis program content, and to be easily understood by the reader, a combination of written review verification and simulation operation verification shall be adopted. Verification is carried out from the aspects of whether the text description is wrong, whether the technical content is complete, whether the information description is consistent with the actual situation, whether the description is easy to understand, whether the operation procedure and method are wrong, whether the technical data are wrong, whether the technical data are coordinated with each other, and whether the warning information is clear and understandable.

\section{Write examples}

\subsection{Writing examples of mechanical fault diagnosis}

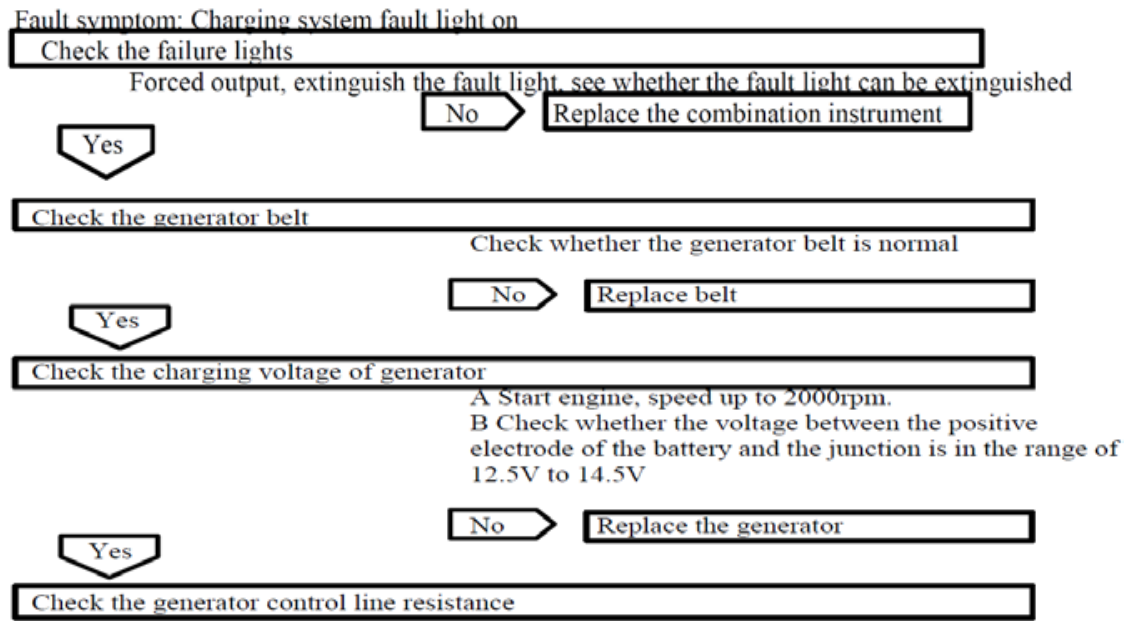

Wire harness connector FA030

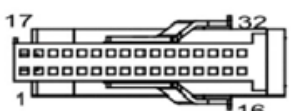

Wire harness connector EM012

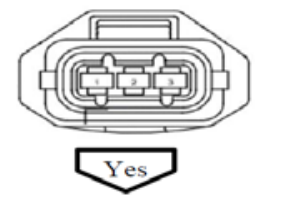

Replace the generator

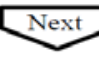

normal work
A Disconnect generator and instrument harness plugins

$\mathrm{B}$ Check the resistance between terminal EM012 of generator wiring harness connector and terminal FA030 of instrument wiring harness connector and ground

C Verify that the measured value is within the specified range

\begin{tabular}{|c|c|c|}
\hline $\begin{array}{l}\text { Measuring } \\
\text { terminal } 1\end{array}$ & $\begin{array}{l}\text { Measuring } \\
\text { terminal } 2\end{array}$ & $\begin{array}{l}\text { Standard } \\
\text { values }\end{array}$ \\
\hline EMO12 (1) & FA030 (6) & $\mathrm{R}<5 \Omega$ \\
\hline EM012 (1) & grounding & $\infty$ \\
\hline
\end{tabular}

A Replace generator, see generator replacement

$B$ Verify that the system is working properly 


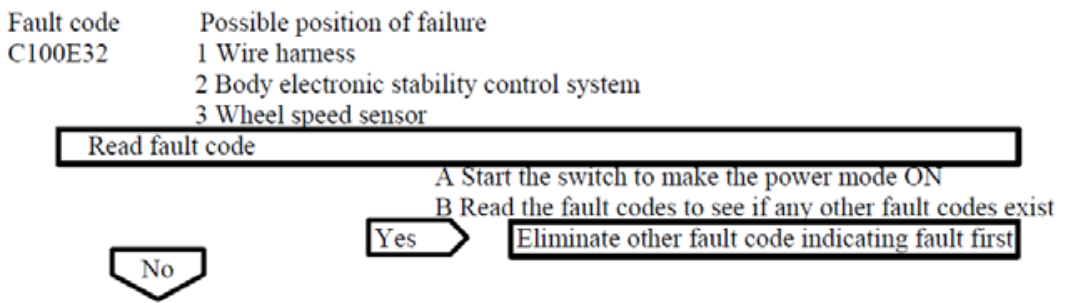

Check the wiring between the body electronic stability control system (ESC) and the right front wheel speed sensor

Wire harness connector CA10B

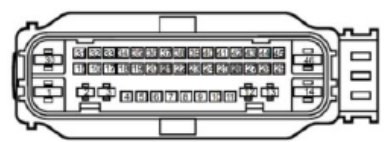

Wire harness connector CA40
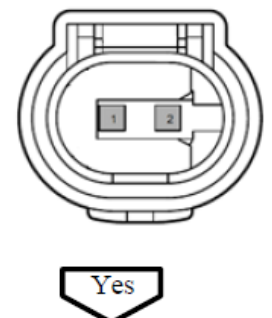

A Start the switch to make the power mode to OFF state

$\mathrm{B}$ Disconnect the ESC harness connector CA10B

$\mathrm{C}$ Disconnect the right front wheel speed sensor wire harness connector CA40

D Check the voltage of ESC harness connector CA10B and CA40 terminals

\begin{tabular}{|l|l|l|}
\hline $\begin{array}{l}\text { Measuring } \\
\text { terminal 1 }\end{array}$ & $\begin{array}{l}\text { Measuring } \\
\text { terminal 2 }\end{array}$ & $\begin{array}{l}\text { Standard } \\
\text { values }\end{array}$ \\
\cline { 1 - 2 } CA10B (26) & CA40 (1) & $\begin{array}{l}\text { Standard } \\
\text { voltage: 0V }\end{array}$ \\
\hline CA10B (21) & CA40 (2) & DAtage \\
\hline
\end{tabular}

E Verify that the measured value is within the specified range

No Replace the wiring harness

Check the circuit between the body electronic stability control system (ESC) and right front wheel speed sensor for a short circuit to the power supply

A Start the switch to make the power mode to OFF state

B Disconnect the ESC harness connector CA10B

$\mathrm{C}$ Disconnect the right front wheel speed sensor wire

harness connector CA40

D Operate the start switch to make the power mode ON

E Check the CA40 terminal and ground resistance

\begin{tabular}{|l|l|l|}
\hline $\begin{array}{l}\text { Measuring } \\
\text { terminal 1 }\end{array}$ & $\begin{array}{l}\text { Measuring } \\
\text { terminal 2 }\end{array}$ & $\begin{array}{l}\text { Standard } \\
\text { values }\end{array}$ \\
\hline CA40 (1) & grounding & Standard \\
CA40 (2) & grounding & $\begin{array}{l}\text { resistance: } \\
10 \mathrm{~K} \Omega \text { or } \\
\text { higher }\end{array}$ \\
\hline
\end{tabular}

F Verify that the measured value is within the specified range

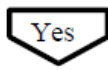

No

Replace the wiring harness

Check circuit to ground short circuit between body electronic stability control system (ESC) and right front wheel speed sensor

A Start the switch to make the power mode to OFF state

$\mathrm{B}$ Disconnect the ESC harness connector CA10B 


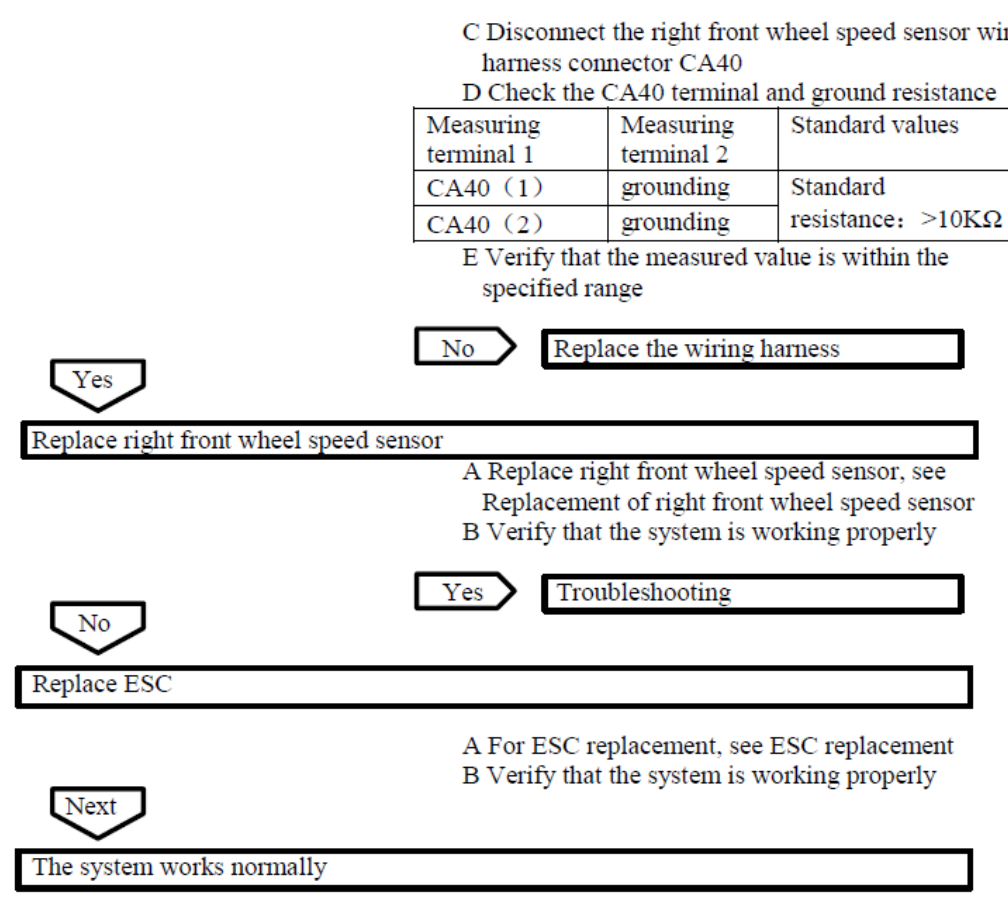

\section{Conclusion}

In this paper, by analyzing the problems and reasons in the compilation of fault diagnosis information, writer summarizes the main contents and compilation methods of the compilation of fault diagnosis information, and gives examples of the compilation of mechanical and electronic fault diagnosis information, so as to provide the compilation ideas and methods for the technicians who compile the fault diagnosis information, and guide them to compile the information that can effectively judge and solve the fault. With the help of fault diagnosis information, according to the logic inference method provided, the success rate of fault solving is improved.

\section{References}

1. Kou. Discussion on Mechanical Fault Diagnosis and Preventive Measures of Automobile Engine[J].AUTO TIME.2020(11):169-170

2. Haibing Li. Analysis of automobile electronic fault detection technology[J]. ELECTRONIC TECHNOLOGY \& SOFTWARE ENGINEERING. 2018(13):240

3. Bulletin of the State Council of the people's Republic of China. Ministry of transport Ministry of Ecology and Environment of the people's Republic of China etc printed and distributed <Management measures for the implementation of automobile maintenance technology information disclosure $>$ Notice[Z].2015(05).

4. Guofang $\mathrm{Wu}$, Fujia Liu etc. Thoughts and methods of compiling automobile maintenance information[C]. Automation and Electrical Engineering, 2020[C]:469-473

5. Bin Xiao. Study on Typical Fault Diagnosis and Troubleshooting Methods of Automobile ESP Based on Diagnostic Flow Chart[J]. INTERNAL COMBUSTION ENGINE \& PARTS. 2021 (06) 